Acta Cryst. (1994). D50, 17-23

\title{
MAD Phasing: Treatment of Dispersive Differences as Isomorphous Replacement Information
}

\author{
By Thomas C. TeRWILliger \\ Mail Stop M880, Life Sciences Division, Los Alamos National Laboratory, Los Alamos, NM 87545, USA
}

(Received 12 Janaury 1993; accepted 3 August 1993)

\begin{abstract}
A framework analogous to that used for the analysis of data in the method of isomorphous replacement is applied to the analysis of multi-wavelength anomalous-diffraction (MAD) data. The present method is advantageous in that refinement of parameters describing the anomalously scattering atoms in the structure can be carried out using nearly all the data, and in that phase information can be readily combined. The procedure described here involves an approximation in which it is assumed that the magnitude of the structure factor corresponding to anomalously scattering atoms in the unit cell is small compared to that corresponding to all other atoms in the structure. A model calculation is applied to a protein crystal with 682 non- $\mathrm{H}$ atoms in the asymmetric unit and two $\mathrm{Se}$ atoms as the anomalous scatterers. It is shown using this model calculation that the approximation used in this analysis does not substantially affect the accuracy of phase calculations for this MAD data. The method is demonstrated by application to MAD data collected on gene $\mathrm{V}$ protein.
\end{abstract}

\section{Introduction}

The multi-wavelength anomalous-diffraction (MAD) technique has now found widespread application to macromolecular structure determination (Karle, 1980; Hendrickson, 1991). In the MAD technique, Bijvoet pairs of structure factors are measured at several X-ray wavelengths, near to and distant from an absorption edge for an atom present at a small number of sites in the asymmetric unit of the crystal. In the method developed by Hendrickson (1991), these structure factors, and the anomalous and dispersive differences between them, are then used in several steps to estimate the structure factors corresponding to all atoms in the structure $\left(\mathbf{F}_{\mathbf{Z}}\right)$. In the first step, structure-factor amplitudes $\left(F_{A}\right)$ corresponding to anomalously scattering atoms in the asymmetric unit are estimated by a least-squares method and are used in a Patterson synthesis to identify the locations of these atoms. The magnitudes of the structure factors corresponding to all atoms in the asymmetric unit $\left(F_{7}\right)$, and the phase differences $(\Delta \varphi)$ between $\mathbf{F}_{\mathrm{A}}$ and $\mathbf{F}_{\mathrm{Z}}$ are also estimated. Next, this step is followed by refinement of parameters describing these atoms against values of $F_{A}$, the estimated structure-factor amplitudes. Finally, the anomalously scattering atom model is used to calculate structure factors for this partial structure. These are used to obtain improved estimates of $\Delta \varphi$ and $F_{Z}$, which lead to the desired structure factors $\mathbf{F}_{\mathbf{Z}}$ (Pähler, Smith \& Hendrickson, 1990). One drawback of this method is the need to use a selected subset of values of $F_{A}$ for refinement of the parameters describing the anomalously scattering atoms (Hendrickson, 1991). In this work, an alternate approach for the analysis of MAD data is developed in which a straightforward approximation is used to convert the data to a form identical to that used in the method of isomorphous replacement. The formulation lends itself to refinement of parameters describing the anomalously scattering partial structure and to calculation of structure factors for the entire structure using previously developed methods.

\section{Methods}

The information obtained from a MAD experiment ordinarily consists of measurements of structurefactor amplitudes for Bijvoet pairs of reflections, $F^{+}\left(\lambda_{j}\right), F^{-}\left(\lambda_{j}\right)$, at several X-ray wavelengths, $\lambda_{j}$. As errors in the elements of a Bijvoet pair are often correlated, and as errors in their differences are not likely to be correlated with their average values, it is convenient to use the average structure-factor amplitude, $\bar{F}\left(\lambda_{j}\right)$, and the Bijvoet differences, $\Delta_{\mathrm{ANO}}\left(\lambda_{j}\right)$, in this analysis. These can be defined as follows,

$$
\begin{gathered}
\bar{F}\left(\lambda_{j}\right)=\frac{1}{2}\left[F^{+}\left(\lambda_{j}\right)+F^{-}\left(\lambda_{j}\right)\right] \\
\Delta_{\mathrm{ANO}}\left(\lambda_{j}\right)=F^{+}\left(\lambda_{j}\right)-F^{-}\left(\lambda_{j}\right) .
\end{gathered}
$$

The ultimate goal of the analysis is to obtain the average structure-factor amplitude, $\bar{F}\left(\lambda_{o}\right)$, and its phase for each reflection at some $\mathrm{X}$-ray wavelength, $\lambda_{o}$. In this approach, however, there will be an intermediate goal of separating the contribution of 
the anomalously scattering atoms to $\bar{F}\left(\lambda_{j}\right)$ from that of all other atoms so as to make it compatible with the method of isomorphous replacement. Let the structure factor from anomalously scattering atoms at X-ray wavelength $\lambda_{j}$ be $\mathbf{F}_{\mathbf{H}}\left(\lambda_{j}\right)$, and the structure factor from all other atoms, independent of X-ray wavelength, be $\mathbf{F}_{\mathbf{o}}$. Then, as in the method of isomorphous replacement, it can be written that

$$
\bar{F}\left(\lambda_{j}\right)=\left|\mathbf{F}_{\mathbf{o}}+\mathbf{F}_{\mathbf{H}}\left(\lambda_{j}\right)\right|,
$$

where $\mathbf{F}_{\mathrm{o}}$ and $\bar{F}\left(\lambda_{j}\right)$ correspond to $\mathbf{F}_{\mathbf{P}}$ and $F_{P H}$, respectively, in the method of isomorphous replacement (Blundell \& Johnson, 1976). The immediate aim of this approach will be to obtain estimates of $F_{o}, \bar{F}\left(\lambda_{o}\right)$, and $\Delta_{\mathrm{ANO}}\left(\lambda_{o}\right)$ at a standard wavelength, $\lambda_{o}$, that can then be analyzed using conventional isomorphous replacement techniques.

A central element in the MAD approach is that, at varying X-ray wavelengths, the structure factor corresponding to anomalously scattering atoms in the unit cell changes in magnitude, but not in phase. In this case,

$$
\mathbf{F}_{\mathbf{H}}\left(\lambda_{j}\right)=\mathbf{F}_{\mathbf{H}}\left(\lambda_{o}\right) \frac{f_{o}+f^{\prime}\left(\lambda_{j}\right)}{f_{o}+f^{\prime}\left(\lambda_{o}\right)},
$$

where $\lambda_{j}$ is any X-ray wavelength and $\lambda_{o}$ is an X-ray wavelength arbitrarily defined as a standard. The real part of the scattering factor for the anomalously scattering atoms at wavelength $\lambda_{j}$ is $f_{o}+f^{\prime}\left(\lambda_{j}\right)$. Note that (4) is only valid for cases in which all the anomalously scattering atoms in the unit cell have identical scattering factors. Based on (4), it can also be written that, to a good approximation (Terwilliger \& Eisenberg, 1987), the anomalous differences at various wavelengths are also related in a simple fashion:

$$
\Delta_{\mathrm{ANO}}\left(\lambda_{j}\right) \approx \Delta_{\mathrm{ANO}}\left(\lambda_{o}\right) \frac{f^{\prime \prime}\left(\lambda_{j}\right)}{f^{\prime \prime}\left(\lambda_{o}\right)},
$$

where $f^{\prime \prime}\left(\lambda_{j}\right)$ is the imaginary part of the scattering factor for the anomalously scattering atoms at wavelength $\lambda_{j}$.

A simple approximation is now used to estimate $F_{o}, \bar{F}\left(\lambda_{o}\right)$ and $\Delta_{\mathrm{ANO}}\left(\lambda_{o}\right)$ from experimental MAD data. If most of the scattering power in the unit cell is from non-anomalously scattering atoms, then (3) can be rewritten in an approximate form in which,

$$
\bar{F}\left(\lambda_{j}\right) \simeq F_{o}+F_{H}\left(\lambda_{j}\right) \cos (\alpha) .
$$

Here $\alpha$ is the phase difference between the structure factors corresponding to non-anomalously and anomalously scattering atoms in the unit cell, $\mathbf{F}_{\mathbf{o}}$ and $\mathbf{F}_{\mathbf{H}}\left(\lambda_{j}\right)$, respectively, at this $\mathrm{X}$-ray wavelength. It is assumed further that this phase difference, $\alpha$, is essentially independent of X-ray wavelength. The effect of these rather crude approximations will be examined below.
Using (5) and (6), it is straightforward to convert MAD data into isomorphous replacement data, as highlighted earlier by Hendrickson (1991) and as carried out by Ramakrishnan, Finch, Graziano, Lee \& Sweet (1993). First, (5) can be used directly to estimate anomalous differences at the standard wavelength using measurements at any other wavelength. Next, estimates of $F_{o}$ and $\bar{F}\left(\lambda_{o}\right)$ can be obtained from average structure-factor amplitudes (1) at any pair of wavelengths $\lambda_{i}$ and $\lambda_{j}$ as follows. Using (4) and (6), the component of $\mathbf{F}_{\mathbf{H}}\left(\lambda_{o}\right)$ along $\mathbf{F}_{\mathbf{o}}$, which we term $\Delta_{\mathrm{ISO}}\left(\lambda_{o}\right)$, can be estimated as,

$$
\begin{aligned}
\Delta_{\mathrm{ISO}}\left(\lambda_{o}\right) & \simeq F_{H}\left(\lambda_{o}\right) \cos (\alpha) \\
& \simeq\left[f_{o}+f^{\prime}\left(\lambda_{o}\right)\right] \frac{\bar{F}\left(\lambda_{i}\right)-\bar{F}\left(\lambda_{j}\right)}{f^{\prime}\left(\lambda_{i}\right)-f^{\prime}\left(\lambda_{j}\right)} .
\end{aligned}
$$

Then this estimate can be used along with the experimental data to obtain the quantities of interest:

$$
F_{o} \simeq \bar{F}\left(\lambda_{j}\right)-\Delta_{\mathrm{ISO}}\left(\lambda_{o}\right) \frac{f_{o}+f^{\prime}\left(\lambda_{j}\right)}{f_{o}+f^{\prime}\left(\lambda_{o}\right)}
$$

and

$$
\bar{F}\left(\lambda_{o}\right)=F_{o}+\Delta_{\mathrm{ISO}}\left(\lambda_{o}\right) .
$$

As a set of MAD data ordinarily contains several observations of each Bijvoet pair, (5)-(9) will usually yield more than one independent estimate of the quantities of interest for a particular reflection. These independent estimates can simply be averaged using weighting factors based on the experimental uncertainties and on (5)-(9).

\section{Results and discussion}

\section{Effects of approximations}

The approach described here for conversion of MAD data to a form similar to that used in the method of isomorphous replacement with anomalous-scattering information relies on a fairly crude approximation. It is assumed that the structure-factor amplitude corresponding to anomalously scattering atoms in the unit cell is small compared to that from all other atoms. The effects of this approximation on the estimates of $F_{o}$ and $\bar{F}\left(\lambda_{o}\right)$, the quantities most affected by this assumption are examined here.

In the accompanying paper on application of a Bayesian approach to analyze MAD data, an idealized set of MAD data and 15 sets of MAD data derived from the ideal set with 'experimental' errors of $1-15 \%$ were constructed (Terwilliger, 1993). Each of these sets of data contained MAD data at three $\mathrm{X}$-ray wavelengths for each of 1763 reflections in a system containing a total of $862 \mathrm{C}, \mathrm{N}$ and $\mathrm{O}$ atoms along with two Se atoms as the anomalously scattering atoms. In the ideal data set, the mean 
structure-factor amplitude corresponding to the Se atoms was 78 , and that corresponding to all other atoms was 280 . In this section, these data sets are used to evaluate the effects of the approximations employed in this work on the accuracy of the results.

Fig. 1 illustrates the effects of our approximations on estimates of $F_{o}$, the structure-factor amplitude corresponding to non-anomalously scattering atoms in the unit cell, made using (7) and (8). In this figure, $F_{P}$ is the actual value of this structure-factor amplitude in the 'ideal' model and $F_{o}$ is the estimate obtained using our approach with data containing negligible $(0.01 \%)$ errors. The vast majority of estimates of $F_{o}$ are close to the ideal values, but for small values of $F_{P}$, some of the estimates of $F_{o}$ are much too small, and some are even negative. This means that even under the best of circumstances, it will not be possible to obtain phase information for every reflection using this approach, but most estimates of $F_{o}$ are accurate.

The quantity that is most seriously affected by the approximations used in (5)-(9) is the estimate of the dispersive difference, $\bar{F}\left(\lambda_{o}\right)-F_{o}$, given by (7) as, $\Delta_{\text {ISO }}\left(\lambda_{o}\right) \simeq F_{H}\left(\lambda_{o}\right) \cos (\alpha)$. Fig. 2(a) illustrates the relationship between the true values of this dispersive difference, $\bar{F}\left(\lambda_{o}\right)-F_{o}$, and those obtained using (7) from data with negligible $(0.01 \%)$ errors, $\Delta_{\mathrm{Iso}}\left(\lambda_{v}\right)$. Once again, the overwhelming majority of estimates of the dispersive difference were close to their ideal values, while a small fraction of the estimates were considerably in error. The distribution of values of $\Delta_{\text {ISo }}\left(\lambda_{o}\right)$ is skewed in Figs. 1 and $2(a)$ because the geometry of the approximation used in (6)-(9)

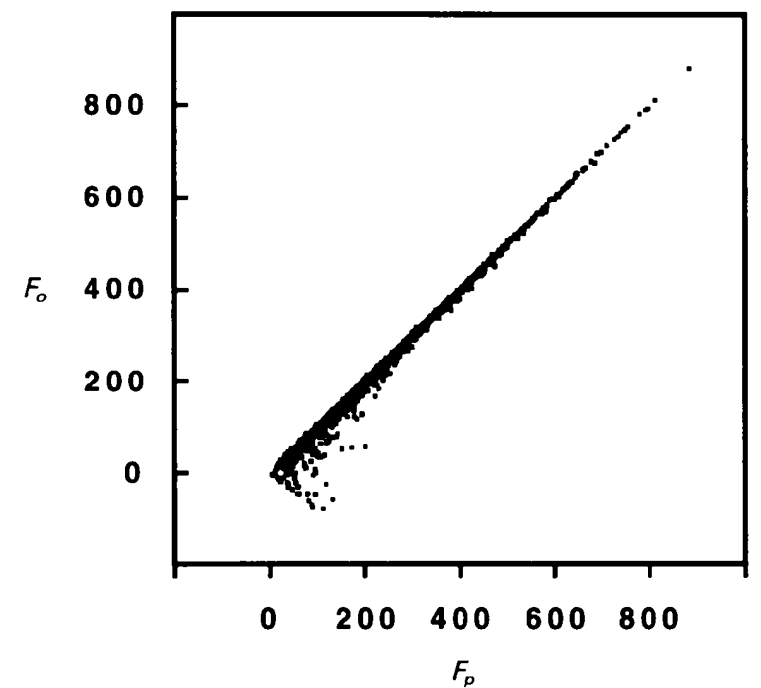

Fig. 1. Effects of approximations on estimates of $F_{o} . F_{P}$ is the actual value of the structure-factor amplitude corresponding to non-anomalously scattering atoms in the 'ideal' model and $F_{o}$ is the estimate obtained using (5)-(9) with data containing $0.01 \%$ errors (see text). always yields $\Delta_{\mathrm{ISO}}\left(\lambda_{o}\right)>\bar{F}\left(\lambda_{o}\right)-F_{o}$ if the data are exact.

The magnitude of the errors introduced into estimates of $\bar{F}\left(\lambda_{o}\right)-F_{o}$, by the approximations used in (5)-(9) can be put into perspective by comparing them with the errors in this quantity introduced by errors in the experimental data itself. In Fig. 2(b), the estimates of $\bar{F}\left(\lambda_{o}\right)-F_{o}$ obtained from data containing $4 \%$ experimental errors are shown as a functions of their ideal values. By comparison of Figs. 2(a) and $2(b)$, it may be seen that the typical errors introduced from the use of the approximations in (5)-(9) are

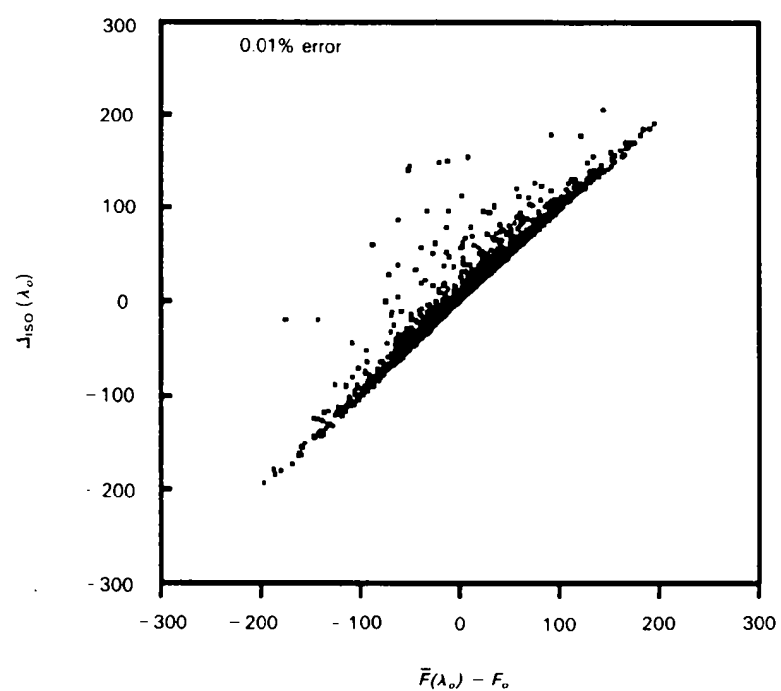

(a)

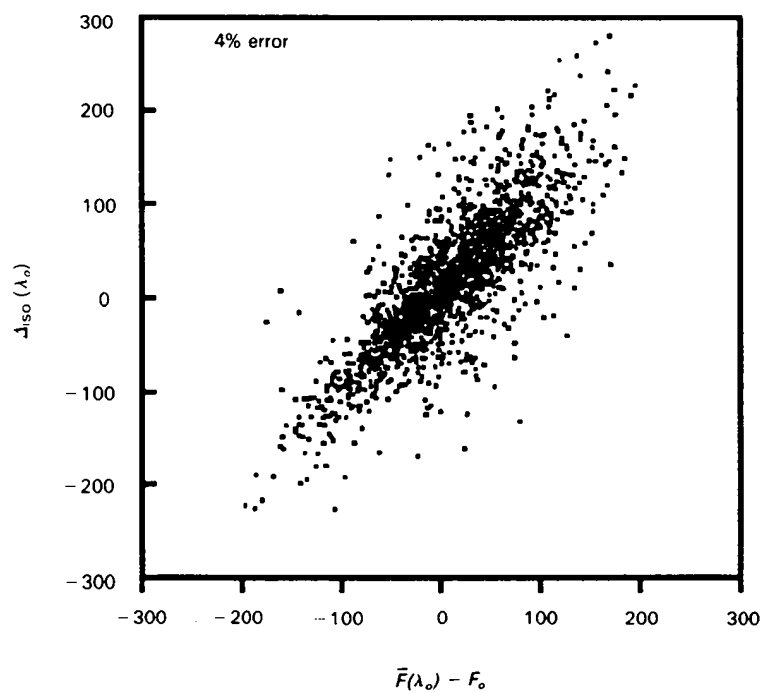

(b)

Fig. 2. Effects of approximations on estimates of $\bar{F}\left(\lambda_{o}\right)-F_{o}$. (a) Dispersive differences obtained using (7) from data with $0.01 \%$ errors, $\Delta_{\mathrm{Iso}}\left(\lambda_{t}\right)$, as a function of their true values in the ideal model, $\bar{F}\left(\lambda_{o}\right)-F_{o}$ (see text). (b) Dispersive differences as in $(a)$, but obtained from data with errors of $4 \%$. 
considerably smaller than those introduced by the experimental error of $4 \%$.

Evaluation of accuracy of refinement of parameters describing anomalously scattering atoms using model data

The model MAD data sets with $1-15 \%$ error were used to evaluate the overall utility of this approach. The MAD data were converted to a form corresponding to that used in the method of isomorphous replacement using (5)-(9). The resulting estimates of $F_{o}, \bar{F}\left(\lambda_{o}\right)$ and $\Delta_{\mathrm{ANO}}\left(\lambda_{o}\right)$ were used in the program HEAVY (Terwilliger \& Eisenberg, 1983, 1987) to refine positional and thermal parameters and to calculate phases for structure factors corresponding to the remainder of the atoms in the model. Both anomalous differences and dispersive differences were included in the refinement and phasing.

Figs. 3(a) and 3(b) illustrate the accuracy of the refinement as a function of the error in the raw MAD data. The occupancies of each of the two Se atoms in the ideal model were each 1.0, and the thermal factors were each $20 \AA^{2}$. For model data

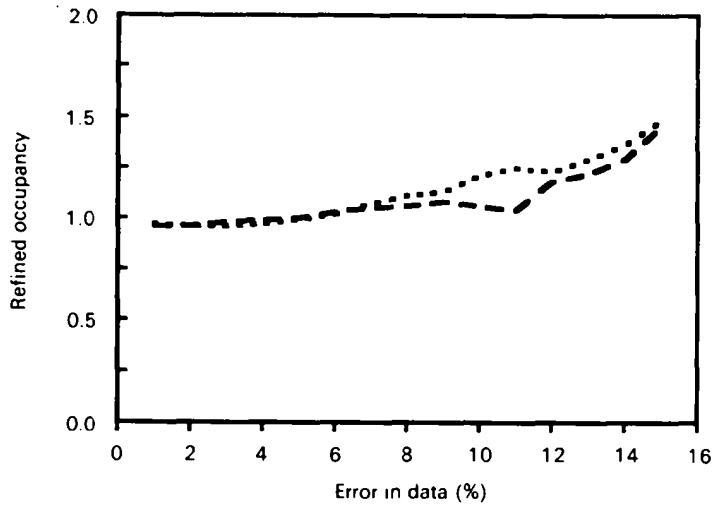

(a)

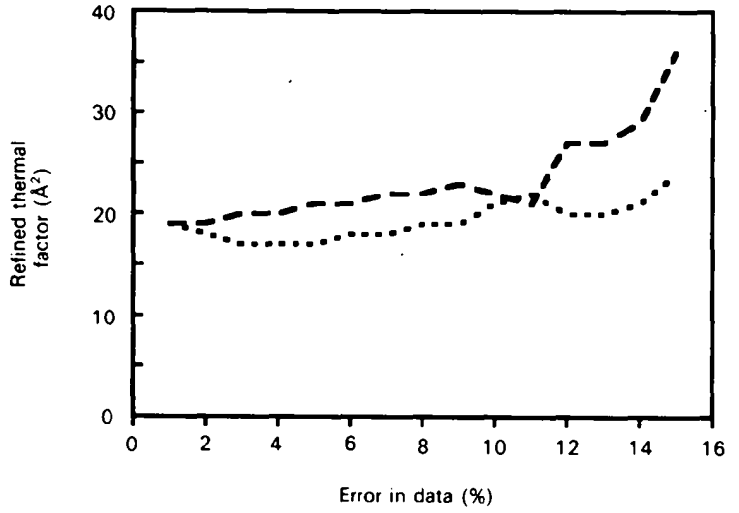

(b)

Fig. 3. (a) Refined estimates of occupancies of the two Se atoms in the model structure as a function of error in the raw MAD data (see text). (b) Refined estimates of thermal factors for the two Se atoms in the model. with errors of up to about $10 \%$, the refined values of both occupancies and thermal factors were close to the ideal values. This indicates that accurate refinement of parameters describing the anomalously scattering atoms can indeed be carried out after conversion of MAD data to a form similar to that used in the method of isomorphous replacement. As discussed above, a small fraction of reflections could not be analyzed using this technique, as estimates of $F_{o}$ were zero or negative for these reflections. This fraction was fairly independent of errors in the experimental data and ranged from 1.8 to $2.2 \%$ of the data over the range of errors of $1-10 \%$.

\section{Combination of MAD data with data from isomor- phous structures}

A very useful feature of the formulation of MAD data presented in (5), (8) and (9) is that, within the limitations imposed by our approximations, the isomorphous and anomalous differences, $\Delta_{\mathrm{ISO}}\left(\lambda_{o}\right)$ and $\Delta_{\mathrm{ANO}}\left(\lambda_{o}\right)$, depend only on the structure factors corresponding to anomalously scattering atoms and their phase angle relative to the structure factors from all other atoms. Furthermore, they depend only weakly on this phase angle, varying as the cosine and sine of this angle, respectively. This means that if two similar structures with the same arrangement of anomalously scattering atoms were considered, the values of $\Delta_{\mathrm{ISO}}\left(\lambda_{o}\right)$ and $\Delta_{\mathrm{ANO}}\left(\lambda_{o}\right)$ for the two structures would be nearly identical even if the structures were not completely isomorphous.

This is useful in several ways. Suppose a very reliable set of structure-factor amplitudes has been measured at a single wavelength on the structure that is of interest. This might be a crystal form isomorphous to the one used for MAD analysis but that does not contain anomalously scattering atoms, for example. Then a pseudo-MIR-with-anomalousscattering data set could be constructed using measured values of $F_{P}$ instead of $F_{o}$ in (9), and with measured values of $\Delta_{\mathrm{ISO}}\left(\lambda_{o}\right)$ and $\Delta_{\mathrm{ANO}}\left(\lambda_{o}\right)$. This mock data set corresponds to the data that would have been obtained had the anomalous scatterers been present in the isomorphous crystal form and had $F_{o}$ values been estimated very accurately. Consequently, using phases and $F_{P}$ structure-factor amplitudes from this mock data set is likely to yield a more accurate electron-density map for the structure of interest than one calculated from the MAD data alone. Note that the 'isomorphous' differences between the MAD and single-wavelength structures are not used in this method. If the structures were very closely isomorphous, then these measured differences could be used as an independent estimate of $\Delta_{\text {ISo }}\left(\lambda_{o}\right)$, but these estimates are likely to generally be less accurate than those from the MAD data which do not suffer from lack of isomorphism. 
A second way to use the 'transportability' of the isomorphous and anomalous differences, $\Delta_{\mathrm{ISO}}\left(\lambda_{0}\right)$ and $\Delta_{\mathrm{ANO}}\left(\lambda_{o}\right)$, is in constructing a MIR data set from several MAD data sets on isomorphous structures differing in the locations of anomalously scattering atoms. In the MIR method, a single value of the 'native' structure-factor amplitude, $F_{P}$, is compared with a number of structure-factor amplitudes corresponding to 'derivatives', $F_{P H}$. If several MAD data sets are collected from different crystals that differ principally in the locations of anomalously scattering atoms, then each data set will have a separate estimate of $F_{o}$. These estimates will ordinarily differ, and even their 'true' values could differ as a result of non-isomorphism among the remainder of the structures. The same approach as described for combining native structure-factor amplitudes with MAD difference data can be applied in this case. An averaged value of $F_{o}$ can be obtained, or a value from single-wavelength data could be used. Then this value can be used in (9) to generate mock values of $F_{P H}$ for each 'derivative' structure. These quantities could then be used in exactly the manner that conventional MIR data is analyzed (Terwilliger \& Eisenberg, 1987). As in the previous example, the isomorphous differences between the structures are not used in this analysis.

Note that the structure factors obtained in this analysis are those corresponding to all atoms except the anomalously scattering atoms, in contrast to the method developed by Hendrickson (1985). The structure factors corresponding to the anomalously scattering atoms can simply be added to those obtained here to obtain a Fourier synthesis for all atoms in the unit cell, if necessary.

\section{Application to actual MAD data collected on gene $V$ protein}

The methods described here were used in the recent structure determination of selenomethioninecontaining gene $\mathrm{V}$ protein (Skinner et al., 1993). Three X-ray diffraction data sets were used in this structure determination, as discussed in the accompanying paper (Terwilliger, 1993). These were a single-wavelength data set on the wild-type protein (not containing selenium), a very good MAD data set on a 'wild-type' protein containing two selenomethionine residues, and a relatively weak and incomplete MAD data set on a mutant protein with three selenomethionine residues. A pseudo-MIR data set was constructed from these three data sets, using measured values of $F_{P}$ from the single-wavelength data set in place of $F_{o}(9)$. The determination of locations of anomalously scattering atoms is described in the accompanying paper (Terwilliger, 1993), and the parameters describing these atoms in the two MAD structures were refined with $H E A V Y$ (Terwilliger \& Eisenberg, 1987) using origin-removed Patterson refinement.

Both anomalous differences and isomorphous differences were used in the refinement of the MAD data. The occupancies of each site refined to values near 0.7 , rather than the value of 1.0 expected, despite the fact that the data had been placed on an approximately absolute scale. The origin of this incomplete occupancy is not clear, as amino-acid analyses of the proteins indicated essentially complete replacement of methionine by selenomethionine. It is possible that this discrepancy is as a result of overestimation of the values of $f^{\prime}$ and $f^{\prime \prime}$, as the values were measured at their extrema, while some variation in wavelength occurred during the course of the X-ray diffraction experiments. In any event, native phases were calculated using the refined parameters for the Se atoms and the pseudo-MIR data, and were used in a Fourier synthesis that was found to be readily interpretable.

As the structure of gene $\mathrm{V}$ protein has now been refined to an $R$ value of $19.2 \%$ at a resolution of $1.8 \AA$, we were able to evaluate the utility of including single-wavelength measurements of $F_{P}$ and of combining phase information from more than one MAD data set on the final accuracy of phases and on the correlation of electron density in the Fourier syntheses with the final model. The results of this comparison are listed in Table 1. Phases calculated using the very weak and incomplete data set on the mutant gene $\mathrm{V}$ protein (I47M) containing three selenomethionine residues in the asymmetric unit, for example, had a mean phase difference from that of the refined model of $67^{\circ}$, and the average electron density in a Fourier synthesis was only 0.59 times the r.m.s. in the map at positions of protein atoms in the refined model. Using single-wavelength measurements of $F_{P}$ in (9) improved the phasing and electron density only very slightly (Table 1 ). The much more complete data set on the 'wild-type' selenomethionine-containing protein had a lower phase difference relative to that of the refined model $\left(62^{\circ}\right)$ and much higher electron density at the positions of protein atoms in the model ( 1.22 times the r.m.s. of the map). Combining the two sets of MAD data and using $F_{o}$ values from the 'wild-type' MAD data set gave a substantial improvement in phase accuracy (56. $2^{\circ}$ difference from the model) and electron density (1.29 times the r.m.s. of the map at positions of protein atoms). Finally, using single-wavelength estimates of $F_{P}$ made little difference in the phase accuracy or electron density. Overall, we conclude that combination of multiple MAD data sets can be very helpful, while including single-wavelength measurements of $F_{P}$ makes only a small difference in phasing accuracy. 
Table 1. Comparison of phases and electron density from combinations of gene $V$ protein $M A D$ and singlewavelength data sets with values based on the refined gene $V$ protein model

Phases and structure-factor amplitudes for the non-anomalously scattering atoms in the unit cell were calculated as described in the text using combinations of MAD data collected on the 'wild-type' and ' $147 \mathrm{M}$ ' mutant gene $\mathrm{V}$ proteins, both containing selenomethione, and using single-wavelength $\mathrm{X}$-ray data $\left(F_{p}\right)$ on a nonselenium-containing wild-type gene $\mathrm{V}$ protein. The combinations used were: $(A)$ I47M MAD data alone; $(B)$ I47M MAD data but using $F_{p}$ instead of $F_{o}$ in (9); (C) 'wild-type' MAD data alone; $(D)$ 'wild-type' MAD data with $F_{r}$ instead of $F_{o}$ in (9); $(E)$ I47M and 'wild-type' MAD data; and $(F)$ I47M and 'wild-type' MAD data with $F_{p}$ instead of $F_{o}$ in (9). Each set of phases is compared with phases calculated from the refined model of gene $V$ protein (Skinner et al., 1993). Each corresponding electron-density map is compared with this refined model by calculating the average value of the electron density at the positions of protein atoms in the model, normalized to the r.m.s. value of the electron density in the unit cell.

$\begin{array}{lccc}\text { Data used } & \text { No. of reflections } & \begin{array}{c}\text { Mesition of atoms in } \\ \text { model } / \sigma\end{array} \\ \text { A I47M } & 1964 & 6 \varphi|\rangle() & \begin{array}{c}\text { pos } \\ B \text { I47M }+F_{r}\end{array} \\ \text { 'Wild type' } & 1950 & 66.4 & 0.59 \\ D \text { 'Wild type' + } F_{r} & 2602 & 62.3 & 0.65 \\ E \text { I47M + 'wild type' } & 2562 & 61.7 & 1.22 \\ F \text { I47M + 'wild type' }+F_{r} & 2610 & 56.2 & 1.22 \\ & 2859 & 56.7 & 1.29 \\ & & & 1.33\end{array}$

Comparison with other methods of MAD data analysis

The most frequently used method of refining parameters describing the occupancies, positions and thermal factors of anomalously scattering atoms in the MAD technique has been to refine them directly against a selected set of $F_{A}$ values obtained from MADLSQ (Hendrickson, 1991). That method has the advantage of using exact expressions to evaluate $F_{A}$ so that in cases with little experimental error or with large structure factors for anomalously scattering atoms the estimates of $F_{A}$ can be very accurate. It has the disadvantage that in cases with substantial errors in measurement, estimates of $F_{A}$ can be much too large and these $F_{A}$ values must be removed from the data set before refinement, leaving only a subset of the data. Once a model for the anomalously scattering atoms has been refined, either the values of $\triangle \varphi$ from MADLSQ (Hendrickson, 1985) can be used to calculate phases directly for the entire structure, or the model can be used to calculate phase probability distributions based on lack-of-closure errors (Pähler et al., 1990).

Kahn et al. (1985) and Ramakrishnan et al. (1993) have described structure determinations in which they converted MAD data to a form compatible with MIR analysis by using data at one wavelength as 'native' data and data at other wavelengths as 'derivative' data. In effect, the 'heavy atoms' of the MIR method consist of the dispersive and anomalous components of the anomalously scattering atoms. The approach of Ramakrishnan et al. (1993) is quite similar to that described here, except that the 'native' structure factors evidently contain the nondispersive part of the structure factors of the anomaloulsy scattering atoms. This means that the 'native' phases are not independent of the 'heavy-atom' phases, and a phase probability distribution slightly modified from that usually used in MIR phasing would be appropriate.

It is important to recognize that the approximations used in the approach described here are not valid when the structure factors corresponding to anomalously scattering atoms are comparable in magnitude to those corresponding to the remainder of the structure. In cases of this type, where the total scattering power of anomalously scattering atoms is similar to that of the other atoms in the unit cell, a treatment of the MAD data with fewer approximations is necessary. The method of Ramakrishnan et al. (1993) is somewhat improved over our method in these cases because, though a similar approximation is made, the approximation only involves the dispersive component of the structure factor corresponding to anomalously scattering atoms, rather than the entire structure factor as in our method. The method of Hendrickson (1985) is likely to be the most accurate in these cases, however, as it does not involve any approximations of this type at all. It is somewhat ironic that in the cases with a strong signal, the method without approximations is likely to be the most accurate, while in cases with a weaker signal methods involving more severe approximation may be more useful.

\section{Concluding remarks}

The simple procedure described here is likely to be of considerable utility in the analysis of MAD data in structures where scattering from anomalously scattering atoms is small compared to that from other atoms in the structure. As the data are cast in a form that is similar to that used in the method of isomorphous replacement, MAD data from several experimental setups or from related crystals can readily be combined with each other or with other forms of phase information. Such an ability to combine phase information has been shown to be useful with MAD data previously (Pähler et al., 1990).

Based on the approach developed here and on that described in the accompanying paper (Terwilliger, 1993), the following procedure is suggested for analysis of macromolecular MAD data with a relatively weak anomalous signal. First, the data is carefully scaled to minimize both dispersive and anomalous differences, as in other methods, and is converted to estimates of average structure-factor amplitudes and anomalous differences at each X-ray 
wavelength using (1) and (2). Next, the procedure described in the accompanying paper (Terwilliger, 1993) that incorporates a Bayesian analysis $(F A B E S T)$ is used to estimate values of $F_{A}$, and these values are used in a Patterson synthesis to identify the locations of anomalous scattering atoms. Additional Patterson syntheses based simply on anomalous differences or on dispersive differences can also be calculated at this point. Finally, the data are converted to a form similar to that used in the method of isomorphous replacement as outlined here using the program $M A D M R G$. Parameters describing the anomalously scattering atoms are refined by correlation of origin-removed Patterson functions and phases for the non-anomalously scattering part of the structure are calculated in a fashion developed for the analysis of isomorphous replacement data using the program $H E A V Y$ (Terwilliger \& Eisenberg, 1987). If a Fourier synthesis for all atoms is desired, the structure factors for non-anomalously scattering atoms may simply be added to those corresponding to the anomalously scattering atoms in the structure.
This work was supported by generous grants from the NIH and from the Laboratory Directed Research and Development Program of Los Alamos National Laboratory, USA.

\section{References}

Blundell, T. L. \& Johnson, L. N. (1976). Protein Crystallography. London: Academic Press.

Hendrickson, W. A. (1985). Trans. Am. Crystallogr. Assoc. 21, $11-21$.

HeNDRICKSON, W. A. (1991). Science, 254, 51-58.

Kahn, R., Fourme, R., Bosshard, R., Chiadmi, M., Risler, J. L., Dideberg, O. \& Wery, J. P. (1985). FEBS Lett. 179, 133-137.

Karle, J. (1980). Int. J. Quantum Chem. 7, 357-367.

Pähler, A., Smith, J. L. \& Hendrickson, W. A. (1990). Acta Cryst. A46, 537-540.

Ramakrishnan, V., Finch, J. T., Graziano, V., Lle, P. L. \& SWEET, R. M. (1993). Nature (London), 362, 219-223.

Skinner, M. M., Zhang, H., Leschnitzer, D. H., Guan, Y., Bellamy, H., Sweet, R. M., Gray, C. W., Konings, R. N. H., WANG, A. H.-J. \& TeRwilliger, T. C. (1993). In preparation.

Ter WILliger, T. C. (1994). Acta Cryst. D50, 11-16.

Terwilliger, T. C. \& EisenberG, D. (1983). Acta Cryst. A39, 813-817.

Terwilliger, T. C. \& Eisenberg, D. (1987). Acta Cryst. A43, 6-13. 